\title{
Document Clustering with Evolutionary Systems through Straight-Line Programs “slp”
}

\author{
José Luis Castillo Sequera, José Raúl Fernández del Castillo Diez, León Gonzalez Sotos
}

Department of Computer Science, University of Alcala, Madrid, Spain.

Email: jluis.castillo@uah.es, joseraul.castillo@uah.es, leon.gonzalez@uah.es

Received May $16^{\text {th }}, 2012$; revised October $16^{\text {th }}, 2012$; accepted October $23^{\text {rd }}, 2012$

\begin{abstract}
In this paper, we show a clustering method supported on evolutionary algorithms with the paradigm of linear genetic programming. "The Straight-Line Programs (slp)", which uses a data structure which will be useful to represent collections of documents. This data structure can be seen as a linear representation of programs, as well as representations in the form of graphs. It has been used as a theoretical model in Computer Algebra, and our purpose is to reuse it in a completely different context. In this case, we apply it to the field of grouping library collections through evolutionary algorithms. We show its efficiency with experimental data we got from traditional library collections.
\end{abstract}

Keywords: Clustering; Genetic Algorithm; Data Mining; Straight Line Programs

\section{Introduction}

Information Retrieval Systems (IRS) are a class of information systems concerned with databases composed of documents processing user queries, in order to facilitate access to relevant information. Many universities and public libraries use IRS to provide access to catalogs books, journals and other documents. In IRS, Cluster Analysis is the generic name for a wide variety of procedures that can be used to create a classification of objects and has been developed and used in many fields from social science to library and information science [1]. Document Clustering has attracted much interest in recent decades due to the relevance of the problem of finding the group that better describes a document.

Genetic Algorithms (GA's) are probabilistic search methods that apply natural selection for finding global optimum solution of optimization problems having feasible solutions [2]. In GA's a population of strings (called chromosomes), which encodes candidate solutions (called individuals) to an optimization problem, evolves toward better solutions. The chromosomes are selected for reproduction under the action of crossover and mutation to form new population. The new population is evaluated and re-used genetic operators up to achieve an optimal solution. Evolution has proven to be a very powerful mechanism in finding good solutions to difficult problems.

These concepts involve the preservation of the characteristics of the best exponents of a generation in the next generation; moreover one can introduce aleatory changes in the new generation composition by means of crossing over and mutation operations. This aleatory component prevents getting stuck into a local maximun from which you can not escape to reach a global maximun. This would represent one of the main advantages of genetic algorithm in opposition to the traditional search methods as the gradient method. Another advantage is its utility for real time applications, in spite of not providing the optimal solution to the problem it provides almost the better solution in a shorter time, including complex problems to solve by traditional methods.

The purpose of clustering is to divide a given group of objects in a number of groups (clusters). Once the optimization criterion is selected, the clustering problem is to provide an efficient algorithm in order to search the space of the all possible classifications and to find one on which the optimization function is minimized. Taking into account the quality of the solutions of GA in different types of fields and problems, it makes perfect sense to try to use it in clustering problems; also the flexibility associated with GA is one important aspect to bear in mind, because with the same genome representation and just by changing the fitness function one can have a different algorithm.

Furthermore, a slp consists of a finite sequence of computational assignments. Each assignment is obtained by applying some function (selected from given set) to a set of arguments that can be variables, constants or precomputed results. The $s l p$ structure can describe complex computable functions using fewer amounts of computa- 
tional resources than GP-trees. The key point for explaining this feature is the ability of $s l p$ 's for reusing previously computed results during the evaluation process. Another advantage with respect to trees is that the $s l p$ structure can describe multivariate functions by selecting a number of assignments as the output set. Hence one single $s l p$ has the same representation capacity as a forest of trees. We study the practical performance of ad-hoc recombination operators for slp's. and we apply the $s l p$ based GP approach to solve clustering of documents.

\section{1. slp Structure}

Straight line programs are commonly used for solving algebraic and geometric problems. An extensive study of the use of slp's in this context can be found in [3]. The formal definition of slp's we provide in this section is taken from [4].

Definition: Let $\left\{f_{1}, \cdots, f_{n}\right\}$ be a set of functions, where for each $i, 1 \leq i \leq n$, each $f_{i}$ has arity $a_{i}$, and let $\left\{t_{1}, \cdots, t_{m}\right\}$ be a set of terminals.

The set satisfies that: $\left\{t_{1}, \cdots, t_{m}\right\}=V \cup C$ with

$V=\left\{x_{1}, \cdots, x_{p}\right\}$ is a finite set of variables and $C=\left\{c_{1}, \cdots, c_{q}\right\}$ is a finite set of constants.

A straight line program (slp) over these functions is a finite sequence of computational instructions

$P=\left\{I_{1}, \cdots, I_{l}\right\}$, where each $k \in\{1, \cdots, l\}$, The number of instructions $l$ is the length of $P$.

$$
u k:=f_{k}\left(\alpha_{1}, \cdots, \alpha_{a_{k}}\right) \quad \alpha_{\mathrm{i}} \in\left\{t_{1}, \cdots, t_{m}\right\}
$$

$u k$ :

$$
=f_{k}\left(\alpha_{1}, \cdots, \alpha_{a_{k}}\right) \alpha_{\mathrm{i}} \in\left\{t_{1}, \cdots, t_{m}\right\} \bigcup\left\{u_{1}, \cdots, u_{k-1}\right\} \text { for } k>1
$$

Usually a slp $P=\left\{I_{1}, \cdots, I_{l}\right\}$ will be identified with the set of variables $u_{i}$ introduced at each instruction, thus $P=\left\{u_{1}, \cdots, u_{l}\right\}$. Each of the nonterms variables $u_{i}$ can be considered as an expression over the set of terminals:

$\left\{t_{1}, \cdots, t_{m}\right\}$ constructed by a sequence of recursive compositions from the set of functions $\left\{f_{1}, \cdots, f_{n}\right\}$.

Following [4] we denote by $s l p$

$\left(\left\{f_{1}, \cdots, f_{n}\right\},\left\{t_{1}, \cdots, t_{m}\right\}\right)$ the set of all slp's over $\left\{f_{1}, \cdots, f_{n}\right\}$ and $\left\{t_{1}, \cdots, t_{m}\right\}$.

Note 1: If we consider a slp $P$ as a program code, for each instruction $I_{i}$, introducing a new variable that is not a term, then the numbers of these variables coincides with the number of instructions as well as the length of $s l p$. Therefore, in the following we will denote a $s l p$ $P=\left\{I_{1}, \cdots, I_{l}\right\}$ as $P=\left\{u_{1}, \cdots, u_{l}\right\}$.

Each of the $u_{i}$ can be considered as a sub-slp on the set of terminals $\left\{t_{1}, \cdots, t_{m}\right\}$ constructed as a sequence of recursive calls to evaluate functions $\left\{f_{1}, \cdots, f_{n}\right\}$.

Example: Let $\boldsymbol{F}$ be the set of basic operations $F=\{$ AND, OR $\}$ and let $T=\left\{d_{1}, d_{2}, \cdots, d_{x}\right\}$ the set of terminals. In this situation all slp $P \in \operatorname{SLP}(F, T)$ that can be defined are polynomials in $D_{x}$ variables with integer coefficients.

$$
\begin{aligned}
P \equiv u_{1} & :=d_{1} \mathrm{AND} d_{2} \\
u_{2} & :=u_{1} \mathrm{OR} d_{5} \\
u_{3} & :=d_{3} \mathrm{AND} d_{4} \\
u_{4} & :=u_{2} \mathrm{OR} u_{3}
\end{aligned}
$$

Then, the function is computed by evaluating the polynomial $u 4$

$$
\left.\left(\begin{array}{lll}
\left(d_{1}\right. & \text { AND } & d_{2}
\end{array}\right) \text { OR } d_{5}\right) \text { OR }\left(d_{3} \text { AND } d_{4}\right)
$$

The next result computes the cardinal of the set $\operatorname{slp}_{l}\left(\left\{f_{1}, \cdots, f_{n}\right\},\left\{t_{1}, \cdots, t_{m}\right\}\right)$, i.e. gives the amount of a given length slp $l$. This amount depends on the cardinal of the sets of functions and terminals.

Note 2: All slp $P=\left\{u_{1}, \cdots, u_{l}\right\}$ on the sets of functions and terminals can be represented as a directed graph $G_{p}=(V, E)$.

The set of vertices is $V=P^{\prime} \bigcup\left\{u_{1}, \cdots, u_{l}\right\}$, where $P^{\prime}$ contains all the terminals involved in computing. The set of edges $E$ is constructed as follows:

For all $k, 1 \leq k \leq l$, draw an edge $\left(u_{k}, a_{i}\right)$ for each $i \in\left\{1, \cdots, a_{j k}\right\}$. Note that $P^{\prime}$ is the set of leaves of the graph $G_{P}$ and is a subset of terminals $\left\{t_{1}, \cdots, t_{m}\right\}$. By example, the next graph shows the directed graph associated with the $s l p$ described in Equation (1) (See Figure 1).

\section{2. slp's versus Trees}

Definition: Let $P=\left\{u_{1}, \cdots, u_{l}\right\}$ a slp on the sets of functions and terminals. For each $k \in\{1, \cdots, l\}$, we define the tree $T_{k}$ associated with non term variable $u_{k}$ as:

Label the root of the tree $T_{k}$ as $f_{j k}$

Label the subtrees of $T_{k}$, from left to right as:

$$
T_{\alpha_{1}}, \cdots, T_{\alpha_{j k}}
$$

In the case that $\alpha_{i}$ is a terminal, this is

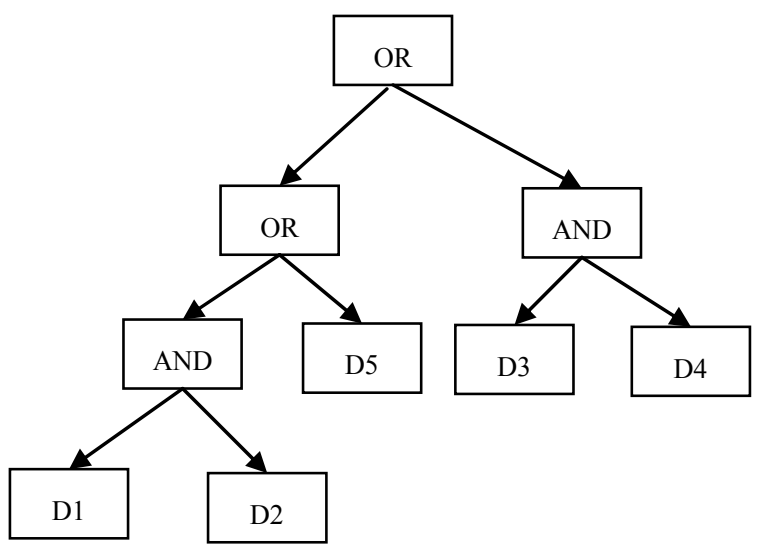

Figure 1. Directed graph associated with the slp. 
$\alpha_{i} \in\left\{t_{1}, \cdots, t_{m}\right\}$ then the tree $T_{\alpha i}$ will have a single node labeled $\alpha_{i}$.

The method for constructing the tree associated with a non-terminal variable $T_{k}$ is a recursive process in each case calculated through subtrees. We can use this property to define a partial order as follows:

Definition: Let $P=\left\{u_{1}, \cdots, u_{l}\right\}$ a slp on the sets of functions and terminals. We define the relation " $<<$ " on it as the one which, for:

$$
u i:=f_{i}\left(\alpha_{1}, \cdots, \alpha_{a i}\right) \quad \text { and } \quad u k:=f_{k}\left(\beta_{1}, \cdots, \beta_{a k}\right),
$$

With $u_{i}<u_{k}$.

If and only if $u i \in\left[\beta_{1}, \cdots, \beta_{a k}\right]$. This relation " $<<$ " is a partial order on the set of programs, in a similar way as the inclusion is a partial order in the power set of a given set.

\subsection{Forest Associated with slp (See Figure 2)}

Let $P=\left\{u_{1}, \cdots, u_{l}\right\}$ a $s l p$ on the sets of functions and terminals. We define the forest associated with $\boldsymbol{P}$ as the set of trees: $\left\{T_{i l}, \cdots, T_{i s}\right\}$, where $M=\left\{T_{i l}, \cdots, T_{i s}\right\}$ is the set of maximal elements of $\boldsymbol{P}$ respect to the partial order «.

Example: Let $P=\left\{u_{1}, \cdots, u_{5}\right\}$ the next $s l p$ on the set of functions $F=\{$ AND,OR $\}$ and terms

$$
\begin{aligned}
T=\left\{d_{1}, d_{2}, d_{3}, d_{4}\right\} & \\
P \equiv u_{1} & :=d_{1} \mathrm{OR} d_{2} \\
u_{2} & :=d_{3} \mathrm{OR} u_{1} \\
u_{3} & :=u_{1} \mathrm{OR} u_{1} \\
u_{4} & :=u_{3} \mathrm{AND} d_{4} \\
u_{5} & :=u_{1} \mathrm{OR} u_{3}
\end{aligned}
$$

Then the set of maximal elements of $\boldsymbol{P}$ respect to the partial order « is $M=\left\{u_{2}, u_{4}, u_{5}\right\}$ and the forest associ- ated with the slp $\boldsymbol{P}$ can be seen in the Figure 3.

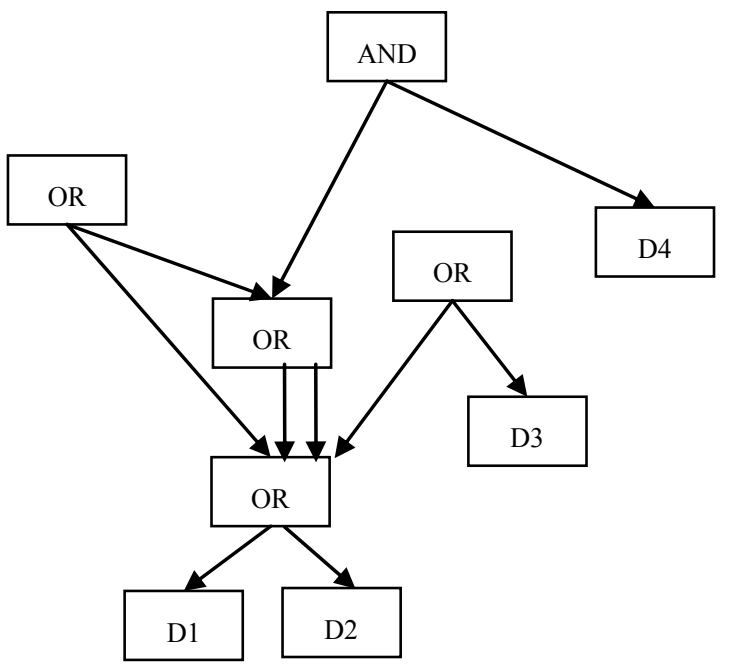

Figure 2. Example of slp with a set of functions and terms.

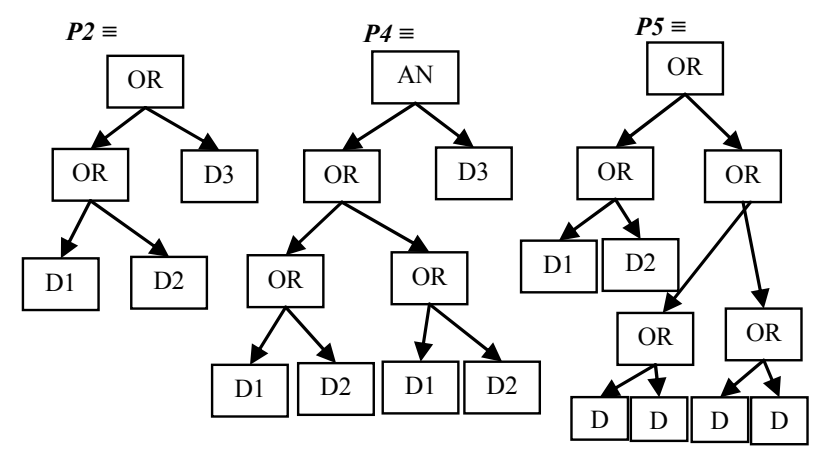

Figure 3. Forest associated with slp P.

\section{Development of Evolutionary System for Document Clustering}

Our evolutionary system makes use of concepts of Genetic Algorithm (GA) [5,6], that allows to increase its potentiality and versatility. In our proposal, an individual is considered as a chromosome that adopts a proper length (depending on the number of documents). Here, the individual is represented by a binary tree structure grouping all documents in its leaves, where a document is more related to some other if they are in a nearest branch. These structures are formed by nodes. In our system, there are terminal and non-terminal nodes connected by edges. The terminal nodes (leaf nodes) are documents.

Each terminal node contains the vector representation of a document (represented by its characteristic vector). Thus, each document in the collection has a single terminal node. Each non-terminal node contains the result of the function of adaptation (fitness) for the two remaining nodes and the coordinates of the centroid of both. We apply the euclidean distance and the Pearson's correlation coefficient simultaneously to evaluate the fitness of our algorithm. This function must be minimized in order to get the solution for the given crossing and mutation operators designed for the problem.

The effects of different mutation and crossover rates had been compared. In this model documents are represented by vector of terms that they are obtained with NZIPF method [7]. We use term vectors, obtained applying a methodology of processing the documents [8] to obtain the best characteristic vectors, selected from the area defined by the use of the Zipf law [9] and the Goffman Point [10,11].

Because we work with evolutionary algorithms, it needs the adjustment of several parameters that make strong effect on the results. Therefore we have to carefully define the experimentation environment [12] and the dataset that we use in our tests.

\subsection{Chromosome Representation}

An individual is a binary tree whose leaves are the docu- 
ments to be grouped. Our chromosome is represented by a binary tree which is evaluated with a fitness based on a combination of distance and similarity measures, operating in pairs of vectors.

We call "global fitness" to fitness that corresponds to the non-terminal top level node (root node) and "partial fitness" the one obtained in the remaining non-terminal nodes. Therefore in the representation there are many "partial fitness" and a single "global fitness" for each individual (each tree).

In practice, we code each of these structures by a string, in which the zeros $(0)$ denote non-terminal nodes and non zeros substrings represent each document.

Documents appears as an avatar (string) arranged between the zeros, which in polish notation describes the tree. The string is traversed in "preorder".

For example, we show two documentary collections, interpreted through a tour in preorder, as represented by strings 000A50420a $\$$ which corresponds to the documents [110,5,4,2,95,221] placed in a certain order (Figure 4), or $000 \& F h 0300152$ which corresponds to the documents $[205,97,42,3,1,5,2]$ placed in a certain order (Figure 4).

For the first document collection (first string): The character " $\mathrm{A}$ " is an avatar that corresponds to the document 110 , the character " 5 " with the document 5 , the character "4" with the document 4 , the character " 2 " to document 2 , the character "a" with the document 95 , the character "\$" with the document 221.

For the second document collection (second string): The " $\&$ " character is an avatar that corresponds to the document 205, the character " $F$ " with the document 97, the character "h" with the document 42 , the character " 3 " with the document 3 , the character " 1 " to document 1 , the character " 5 " with the document 5 and the character " 2 " with the document 2.

Each of the strings is an individual, whose representa- tion we can show in Figure 4.

\subsection{Initial Population}

Let $F=\left\{f_{1}, \cdots, f_{n}\right\}$ a set of functions such that $f_{i}$ has arity $a_{i}$ for all $i, 1 \leq i \leq n$ and let $T=\left\{t_{1}, \cdots, t_{m}\right\}$ a set of terms as we introduced before. We describe the process for generating each of the $s l p$ initial population.

For the first non-terminal variable $\mathrm{u}_{1}$, select a function $f_{j 12} \in F$ randomly. For each argument $i \in\left\{1, \cdots, a_{j 1}\right\}$ this function $f_{j l}$ randomly choose an element $\alpha_{i}$ from the set of terms $T$. In general, for non-term variable $u_{k}, k>1$, also started choosing a function $f_{j k} \in F$ (from the set of given functions). The difference is now, for each argument $i, 1 \leq i \leq a_{j k}$, choose at random an element in the set $T \bigcup\left\{u_{1}, \cdots, u_{k-l}\right\}$.

In practice, we must introduce an upper bound $L$ for the length of the $s l p$ involved in the process of genetic programming. Therefore, the first step in the generation of each slp should consist of randomly selecting the length $l \in\{1, \cdots, L\}$.

We have fixed 50 as the population's size, so we have 50 different trees for each test. We generate the individuals (chromosomes) in a random way. These chromosomes are called initial population that feed into genetic operator process $[2,5]$. The length of a chromosome depends on the number of documents in the documental database. Genetic operators generate a new population from the existing one, placing similar documents into a given groups (Figure 5). We should have all documents in our documental data base without repetition. This representation of the chromosome is a necessary condition for the algorithm.

\subsection{Fitness Evaluation}

Each individual has a "global fitness" and each node has a "partial fitness". We use two measures of the fitness

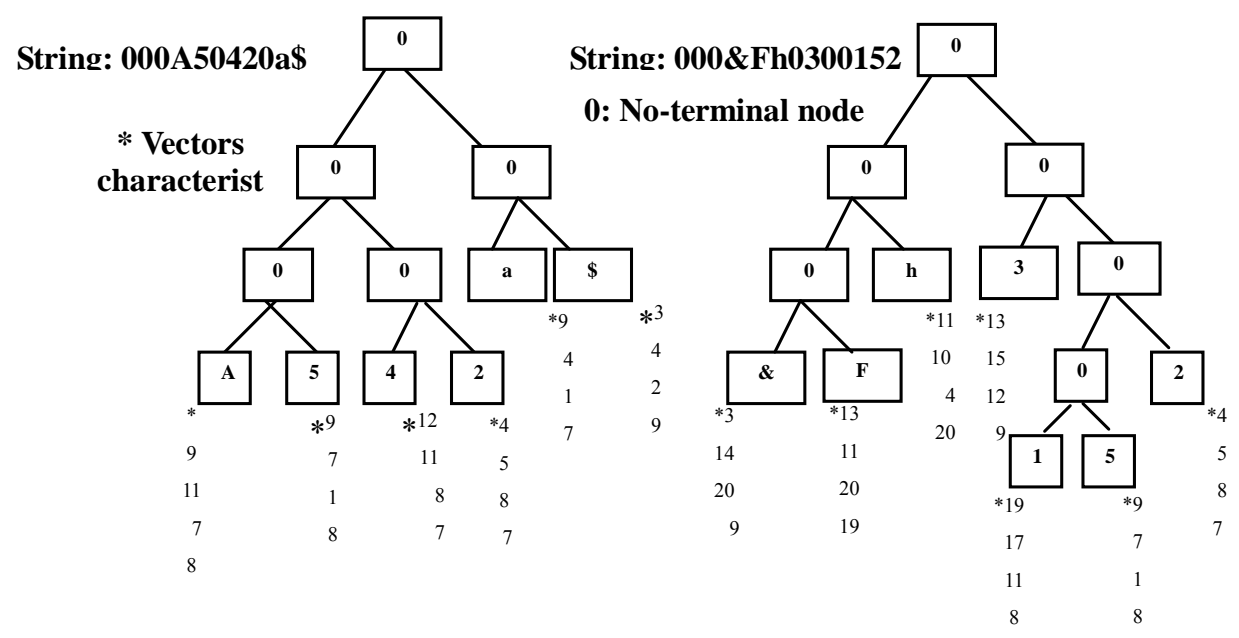

Figure 4. Tree representation of individuals in the system. 


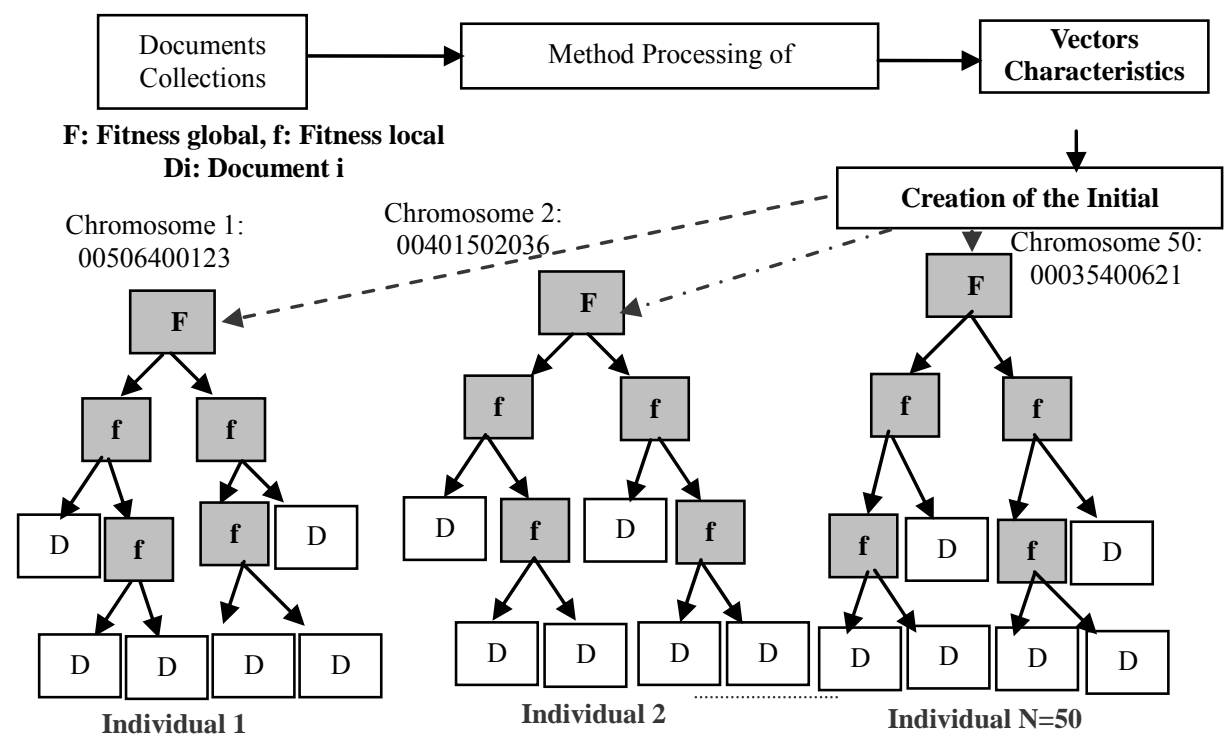

Figure 5: Initial population of individuals.

function to calculate the distance and similarity between documents and to be able to form better clusters. Thus, the proposed fitness on the one hand try to maximize the similarity of documents and minimizing the distances between them, (see Table 1). where the $\alpha$ coefficient is the weight that balances the values of the distance (Euclidean) and of the value of similarity ( $\alpha$ varies between 0 and 1), ie the coefficient of the convex combination of the distance and the inverse of the similarity, and each $d_{i}$ is represented by its characteristic vector.

\subsection{Selection}

After we evaluate population's fitness, the next step is chromosome selection. Selection embodies the principle of 'survival of the fittest'. Satisfied fitness chromosomes are selected for reproduction, for it, we apply the method of selection of the tournament, using a tournament of 2 , and we apply Elitism in each generation using the strategy called GAVAPS (Genetic variation in the size of the population) $[2,13]$ using the concept of age and time of life, so that if an individual has better fitness will have more time to live. Therefore, this approach keeps elitism in subsequent generations.

\subsection{Genetic Operator}

With the slp data structure can be implemented genetic operators used in the field of evolutionary computing, allowing its full potential, such as:

\subsubsection{Propossed Crossover Operator with the Structure slp}

As slp is coded as a finite sequence of operations and individuals have the same length, classical crossover methods, such as cross at one point, two point crossover and the uniform crossover can be adapted to this data structure. Here are the details:

Definition: Let $P_{1}=\left\{u_{1}, \cdots, u_{L}\right\}$ and $P_{2}=\left\{u_{1}^{\prime}, \cdots, u_{L}^{\prime}\right\}$ two slp with the same number of functions and terms. Then, the above crossover operators, act on these structures as follows:

At one point: Since breaking point $i \in\{1, \cdots, L\}$, crossing at a point operating on the slp $P_{1}$ and $P_{2}$ produces:

$$
\begin{aligned}
& P_{1}^{\prime}=\left\{u_{1}, \cdots, u_{i}, u_{i+1}^{\prime}, \cdots, u_{L}^{\prime}\right\} \\
& P_{2}^{\prime}=\left\{u_{1}^{\prime}, \cdots, u_{i}^{\prime}, u_{i+1}, \cdots, u_{L}\right\}
\end{aligned}
$$

At two points: Given two break points $i, j \in\{1, \cdots, L\}$ with $i<j$ crossing at two points operating on the $\operatorname{slp} P_{1}$ and $P_{2}$ produces:

$$
\begin{aligned}
& P_{1}^{\prime}=\left\{u_{1}, \cdots, u_{i}, u_{i+1}^{\prime}, \cdots, u_{j}^{\prime}, u_{j+1}, \cdots, u_{L}\right\} \\
& P_{2}^{\prime}=\left\{u_{1}^{\prime}, \cdots, u_{i}^{\prime}, u_{i+1}, \cdots, u_{j}, u_{j+1}^{\prime}, \cdots, u_{L}^{\prime}\right\}
\end{aligned}
$$

Uniform: Given a Boolean vector $\alpha=\left\{\alpha_{1}, \cdots, \alpha_{L}\right\} \in\{0,1\}^{L} \quad$ uniform cross operating on the slp $P_{1}$ and $P_{2}$ produces:

$$
P_{1}^{\prime}=\left\{v_{1}, \cdots, v_{L}\right\}, P_{2}^{\prime}=\left\{v_{1}^{\prime}, \cdots, v_{L}^{\prime}\right\}
$$

where for all $i \in\{1, \cdots, L\}$ holds:

$$
\begin{aligned}
& \text { If } \alpha_{i}=0 \text { then } v_{i}=u_{i} \text { and } v_{i}^{\prime}=u_{i}^{\prime} \\
& \alpha_{i}=1 \text { then } v_{i}=u_{i}^{\prime} \text { and } v_{i}^{\prime}=u_{i}
\end{aligned}
$$

Although it is possible to apply any of the discussed crossover operators with slp structure, we had to develop a new crossover operator to avoid the possibility of having a given documents twice in an individual. 


\subsubsection{Crossover Operator Used}

We developed a crossover operator based on a crossover mask, which applies on the populations of individuals. First we selected two parent individuals by the method of the tournament, then we choose randomly one chromosome and this chromosome is used to define the mask for the crossing. We call the "parent mask chosen", the individual chromosome randomly chosen and "un chosen parent mask" the remaining one.

At the beginning the new individual will have as avatar the chromosome of the chosen parent mask. Then, the crossing is made by examining the chromosomes gene by gene from both parents. If genes are a non-terminal node (node 0), we keep the gene that corresponds to the mask of the father elected to the new individual child. But if we find terminal nodes (documents) in both parents' genes on the chromosomes, then we select the gene for the mask of the unselected parent, and this gene is sought and exchanged in the new individual with a corresponding gene of the mask of unelected father $[14,15]$. For example, if we have 5 documents and we have the following chromosomes parents:

$$
\begin{array}{lllllllll}
0 & 0 & 2 & 1 & 0 & 5 & 0 & 3 & 4 \\
0 & 0 & 0 & 5 & 3 & 0 & 2 & 1 & 4
\end{array}
$$

The chromosome created after applying the crossing operator would be:

$$
\begin{array}{lllllllll}
0 & 0 & 0 & 1 & 3 & 0 & 2 & 5 & 4
\end{array}
$$

Then, the new individual will always have documents that are not repeated (Figure 6).

\subsubsection{Mutation Operator}

The mutation operator is asexual and therefore acts on a single parent, making small random changes what will help to maintain diversity in the population that, otherwise, could tend to converge prematurely to a local optimum.

Let $F=\left\{f_{1}, \cdots, f_{n}\right\}$ be a set of functions such that $f_{i}$ has arity $a_{i}$ for all $i, 1 \leq i \leq n$ and let $T=\left\{t_{1}, \cdots, t_{m}\right\}$ be a set of terms as we introduced before. The first step in applying the mutation $s l p$ to $s l p \boldsymbol{P}$ is to select a non-terms variable $u_{i} \in \boldsymbol{P}$ randomly. After than, we select randomly, one of the arguments of the function $f \in$ functions non-terms variable $u_{i}$. For the final step, we replace the argument by one from the set $T \bigcup\left\{u_{1}, \cdots, u_{i-l}\right\}$ chosen randomly. Formally, the slp mutation operation is described:

Let $P=\left\{u_{1}, \cdots, u_{L}\right\}$ a slp on the set of functions and terms and let $u_{i}=f\left\{\alpha_{1}, \cdots, \alpha_{n}\right\}$ the variable non-terminal selected to be mutated, where $f \in$ functions and $\alpha_{k} \in T \bigcup\left\{u_{1}, \cdots, u_{i-l}\right\}$.

Mutation of the slp $\boldsymbol{P}$ at point $i$ occurs: $P^{\prime}=\left\{u_{1}, \cdots, u_{i-1}, u_{i}^{\prime}, u_{i+1}, \cdots, u_{L}\right\}$, where

$$
\begin{aligned}
& u_{i}^{\prime}=f\left(\alpha_{1}, \cdots \alpha_{j-1}, \alpha_{j}^{\prime}, \alpha_{j+1}, \cdots, \alpha_{n}\right), \\
& \alpha_{j} \in T \bigcup\left\{u_{1}, \cdots, u_{i-1}\right\} /\left\{\alpha_{j}^{\prime}\right\}
\end{aligned}
$$

and both $j$ and $\alpha_{j}^{\prime}$ are randomly selected.

Therefore, we use a mutation operator on nodes. This operator is implemented by selecting an individual in the population by the so-called method of the tournament, then randomly selects a pair of nodes of that individual. (If any of them is a terminal node). Then these nodes are exchanged, thus generating a new individual who could have a different tree structure. The new individual has the same base documents but placed in a different order. The mutation involves the alteration of the values of the chromosome depending on a rate of probability. Examples of the use of this operator are depicted in Figures 7 and 8 respectively.

\subsection{Stop Criteria}

Stop criteria is used to determine when the algorithm stops. When the fitness value has no changes during a given number of generations we consider that we got a good individual, representing relations between documents. In any case the maximum number of generations is set to 4000 .

\section{Experiments and Results}

For the real tests we have used documents from the "Reuters 21578" collection [16], taking the distributions with greater dispersion of data (distribution 21, distribution 2, distribution 20, distribution 8) and documents of the collection of editorials of the Spanish newspaper "El Mundo".

Reuters 21578 is a documentary collection, one of the "de facto" standards within the domain of automatic categorization of documents, used by numerous authors. We have also used the collection in Spanish of the editorials of the newspaper "El Mundo" from the years 2006 and 2007, which has been classified manually into the document processing stage. Thus from both collections have taken representative samples of groups of documents. To verify the validity of our work, we used the distributions with higher dispersion. The comparative data is presented in Table 2.

Samples were taken by selecting the documents from two categories of each documental collection, taking those categories that contain the greater quantity in documents. For the collection of the editorials of the newspaper "El Mundo", we apply the same approach, using the main categories that it has the thesaurus Eurovoc [17]. It was necessary to carry out a manual classification previously in each one of the editorials of the collection (1402 editorials). The Table 3 sample ordered the results obtained with the collection of the editorials alphabetically. 
Table 1. Measures of the function.

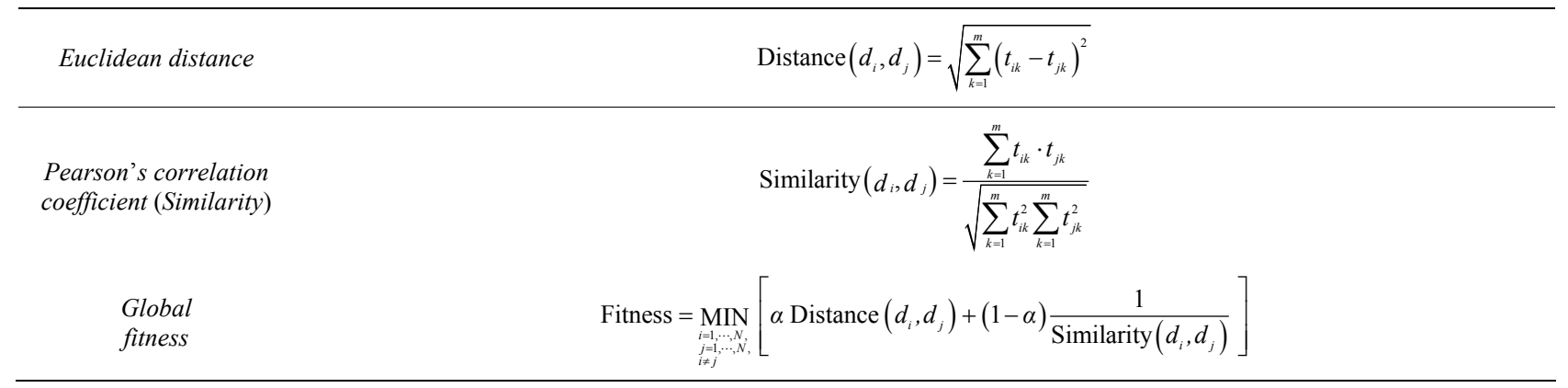

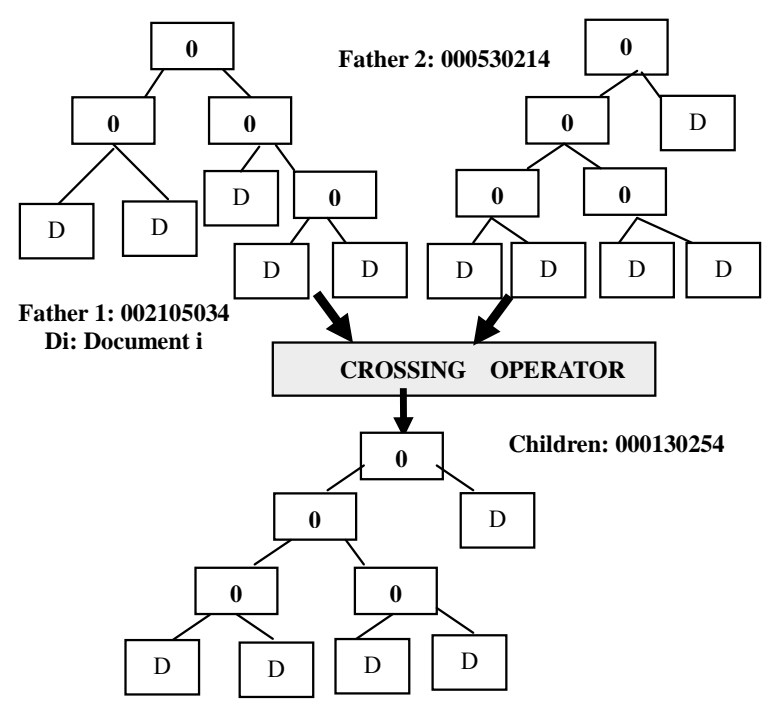

Figure 6. Operator of crossing of GA based on crossing mask.

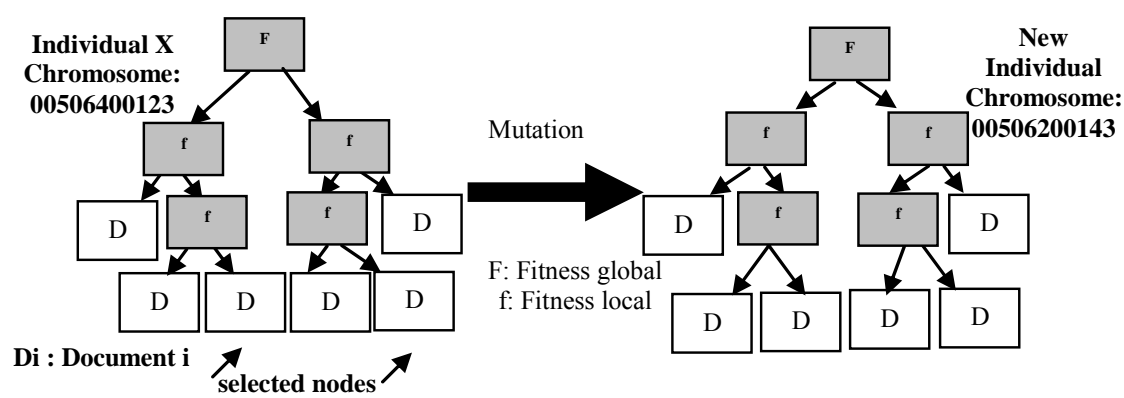

Figure 7. Mutation operator between terminal nodes.

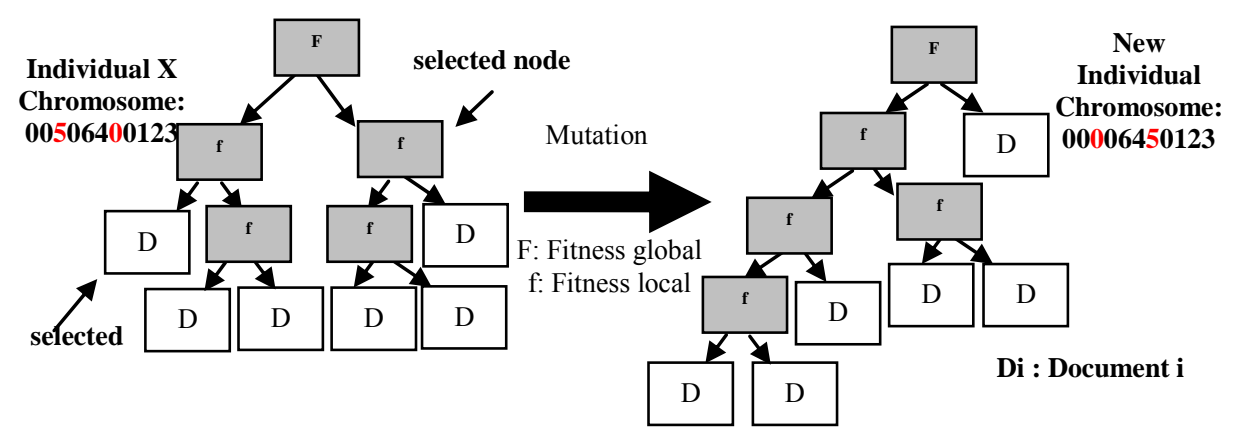

Figure 8. Mutation operator between terminal and nonterminal node. 
Table 2. Rates of dispersion of the distributions "Reuters 21578”.

\begin{tabular}{cccc}
\hline Distribución & Documents & Groups & Dispersión \\
\hline Reuters2-000.SGML & 406 & 31 & 13.09 \\
Reuters2-001.SGML & 436 & 34 & 12.82 \\
Reuters2-002.SGML & $\mathbf{4 5 5}$ & $\mathbf{2 7}$ & $\mathbf{1 6 . 8 5}$ \\
Reuters2-003.SGML & 450 & 32 & 14.06 \\
Reuters2-004.SGML & 446 & 33 & 13.51 \\
Reuters2-005.SGML & 498 & 36 & 13.83 \\
Reuters2-006.SGML & 511 & 38 & 13.44 \\
Reuters2-007.SGML & 475 & 35 & 13.57 \\
Reuters2-008.SGML & $\mathbf{4 5 2}$ & $\mathbf{3 2}$ & $\mathbf{1 4 . 1 2}$ \\
Reuters2-009.SGML & 457 & 33 & 13.84 \\
Reuters2-010.SGML & 425 & 31 & 13.70 \\
Reuters2-011.SGML & 485 & 39 & 12.43 \\
Reuters2-012.SGML & 469 & 37 & 12.67 \\
Reuters2-013.SGML & 199 & 29 & 6.86 \\
Reuters2-014.SGML & 179 & 27 & 6.62 \\
Reuters2-015.SGML & 441 & 35 & 12.60 \\
Reuters2-016.SGML & 488 & 39 & 12.51 \\
Reuters2-017.SGML & 398 & 41 & 9.70 \\
Reuters2-018.SGML & 328 & 39 & 8.41 \\
Reuters2-019.SGML & 316 & 32 & 9.87 \\
Reuters2-020.SGML & $\mathbf{4 0 2}$ & $\mathbf{2 6}$ & $\mathbf{1 5 . 4 6}$ \\
Reuters2-021.SGML & $\mathbf{2 7 3}$ & $\mathbf{1 6}$ & $\mathbf{1 7 . 0 6}$ \\
\hline
\end{tabular}

Table 3. Number of documents in the collection of editorials from the newspaper "El Mundo" grouped by categories of Eurovoc, following the manual classification performed in the film processing stage.

\begin{tabular}{cc}
\hline Eurovoc Category & Number of Documents \\
\hline Agriculture, Forestry and Fisheries & 2 \\
Finance & 9 \\
Social Affairs & 161 \\
Science & 3 \\
European Communities & 22 \\
Law & 302 \\
Education and Communication & 30 \\
Enterprise and Competition & 8 \\
Energy & 20 \\
Geography & 4 \\
Industry & 19 \\
Environment & 5 \\
Economic Exchanges and Trade & 11 \\
International Organizations & 9 \\
Production, Technology and Research & 1 \\
International Relations & 219 \\
Labor and Employment & 13 \\
Transportation & 18 \\
Economic Life & 36 \\
Political Life & 510 \\
\hline
\end{tabular}

Thus, the experiments with the GA, making five executions were carried out on each of the samples taken from experimental collections. The output of the experiment is the best fitness obtained and the convergence speed or the generation where the best fitness was gotten.

Finally, as a measure of the quality of the algorithm is able to serve the best solution obtained and the robustness, i.e. the average quality of the algorithm (average values of the various solutions obtained) [18].

In experiments in our work environment, we used samples of documents collected randomly from "very few (20), few (50), many (80) and enough (150)" documents, with the requirement that belonged to only two categories of Reuters or distribution of Editorials in Spanish. Each sample was processed with 5 different seeds, and each of the results was compared with a result from Kmeans method.

Because of its influence during the experiments, the choice of GA parameters has been analyzed in detail. We attended, the evolution of successes (groups assigned to each document which accords with the distribution), and the changing role of adaptation "fitness" used.

\subsection{Determination of the Value of $\alpha$ in the GA}

To determine this value of $\alpha$, we use the distribution that has the highest dispersion of documents in the Reuters collection (distribution 21) and apply the GA varying the value of $\alpha$ in each of the tests with the usual parameters, always trying to test the effectiveness of the GA. We analyzed the relationship between fitness and the value of $\alpha$ (Figure 9). In Table 4, we show the results in determining the value of $\alpha$ with different samples of documents examined.

The results suggest that a value of $\alpha$ close to 0.85 provides better results because it gives us more effectiveness in terms of number of hits, and a better fitness for the algorithm. This is corroborated with other distribution of the collection.

\subsection{Tests to Determine the Rate for Mutation and Crossover Operators}

We began with an analysis of the system behaviour for different rate of the mutation operator in a wide range of values to cover all possible cases. Thus, for the rate of mutation operator in the range of: $0.01,0.03,0.05,0.07$, $0.1,0.3,0,5,0.7$; that allows us to apply the mutation operator of GA in different circumstances and study their behaviour. To determine the optimal value of the crossover operator over an interval from 0.70 to 0.95 ; selecting high values to apply more frequently the crossing operator for the GA. As an index of the quality of the operator we have the number of hits of the GA. In Figure 10, we show the average number of hits for samples 


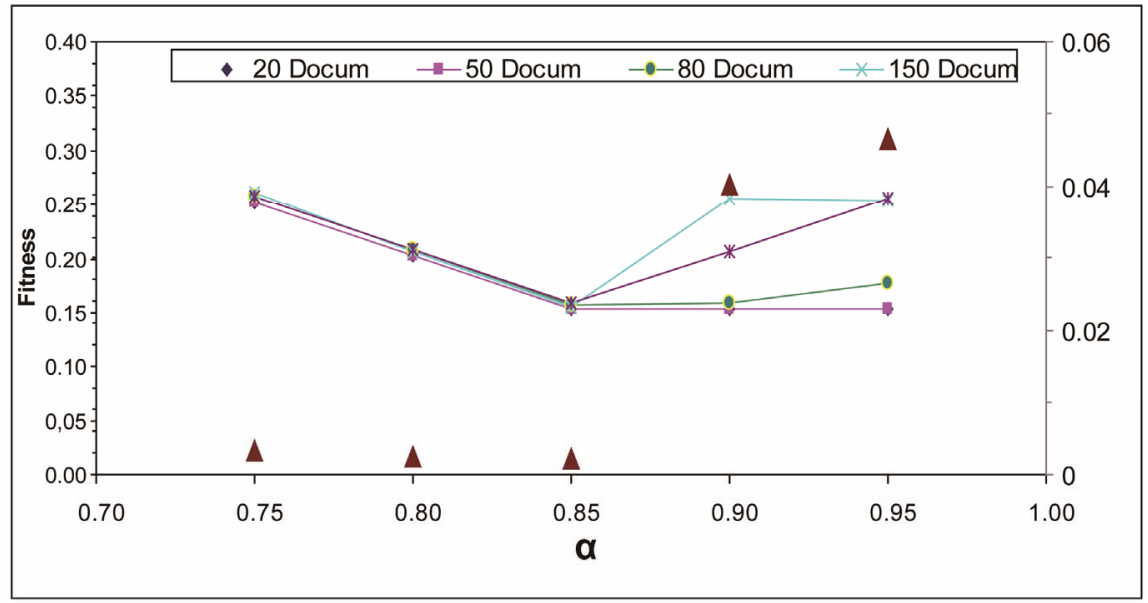

Figure 9. Best Fitness versus $\alpha$ for different samples of documents from the Reuters Collection: Distribution 21. One can see that there is an increased dispersion of fitness values over 0.85 due to the increased contribution of Euclidean Distance which makes it insensitive to the Fitness to find the clusters.

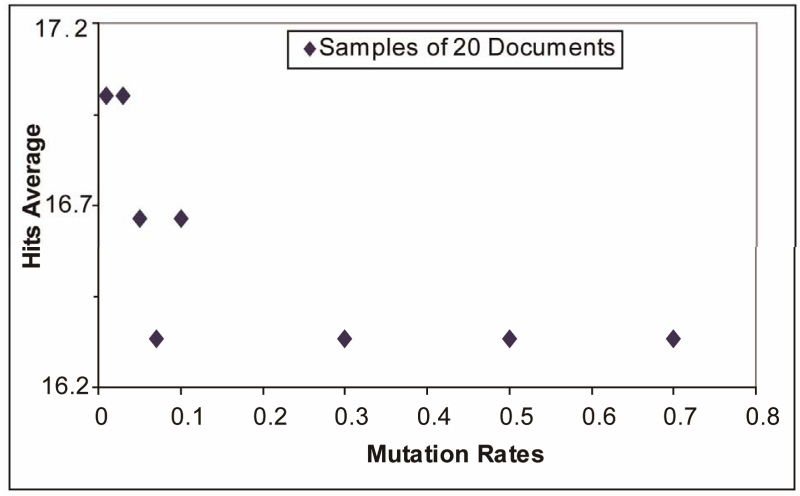

(a)

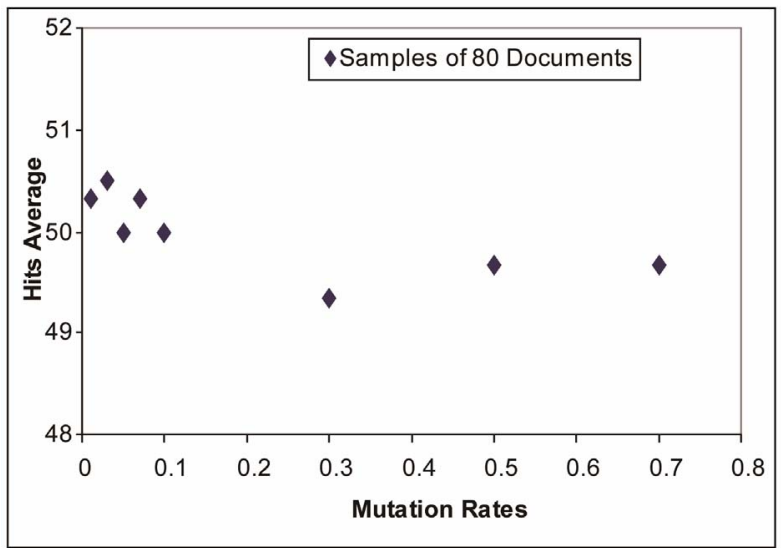

(c)

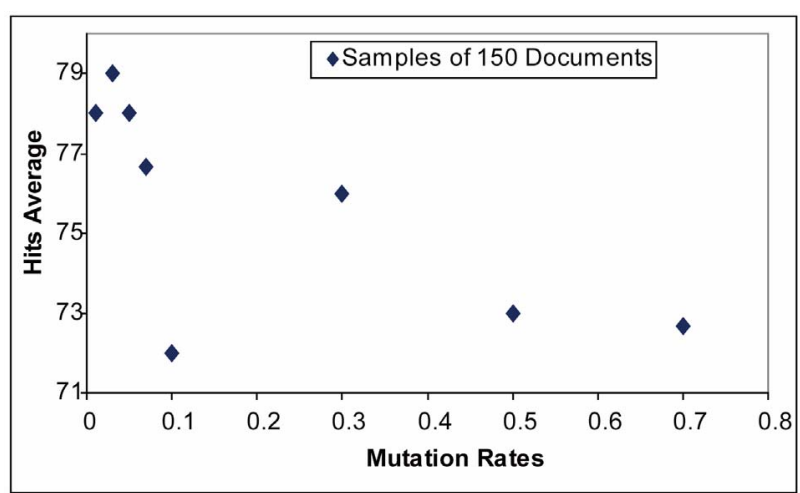

(b)

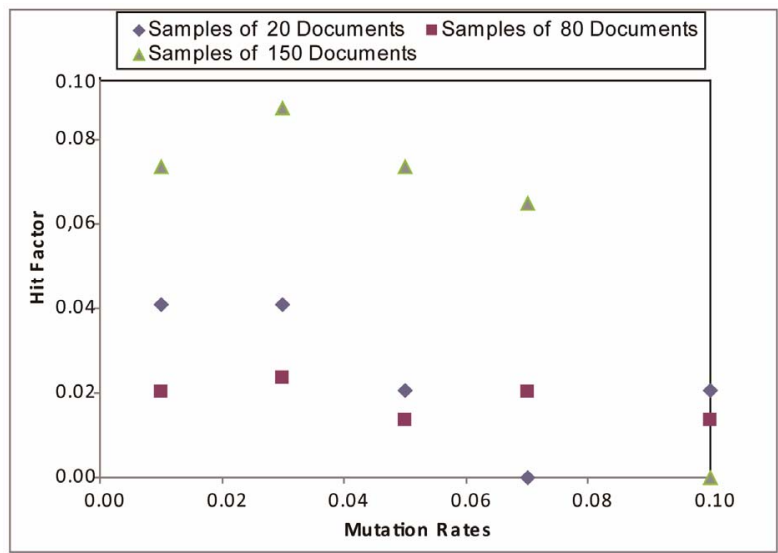

(d)

Figure 10. Hits average of GA with samples 20, 80 and 150 documents varying mutation rate and hit the GA factor against the mutation rates.

of 20, 80 and 150 documents for different mutation rates.

To help in the evaluation of the experimental results we define the hit factor as follows:

$$
a_{i}-a_{0}
$$

where $a_{i}$ is the average number of hits obtained by the GA and $a_{0}$ is the minimum average of hits obtained by the GA.

After experiments we conclude that the best mutation 
Table 4. Results of tests with the GA, taking different samples of documents with the distribution 21 of the Reuters collection, to determine the best value for $\alpha$.

\begin{tabular}{|c|c|c|c|c|c|c|}
\hline Documents & $\alpha$ & Generation & Best Fitness & Average Fitness & Hits & Effectiveness (\%) \\
\hline 20 & 0.75 & 1436 & 0.25291551 & 0.46489675 & 15 & 75.0 \\
\hline 20 & 0.80 & 1592 & 0.20298477 & 0.47026890 & 16 & 80.0 \\
\hline 20 & 0.85 & 2050 & 0.15255487 & 0.24504483 & 17 & 85.0 \\
\hline 20 & 0.90 & 3694 & 0.15266796 & 0.25909582 & 17 & 85.0 \\
\hline 20 & 0.95 & 1520 & 0.15319261 & 0.24596829 & 17 & 85.0 \\
\hline 50 & 0.75 & 3476 & 0.25290429 & 0.28744261 & 35 & 70.0 \\
\hline 50 & 0.80 & 3492 & 0.20285265 & 0.27862528 & 36 & 72.0 \\
\hline 50 & 0.85 & 3355 & 0.15312467 & 0.29128428 & 36 & 72.0 \\
\hline 50 & 0.90 & 2256 & 0.15318358 & 0.28347470 & 36 & 72.0 \\
\hline 50 & 0.95 & 2222 & 0.15345986 & 0.27863789 & 36 & 72.0 \\
\hline 80 & 0.75 & 3049 & 0.25704660 & 0.36871676 & 61 & 76.2 \\
\hline 80 & 0.80 & 1371 & 0.20782096 & 0.33303315 & 61 & 76.2 \\
\hline 80 & 0.85 & 2131 & 0.15784449 & 0.34447947 & 62 & 77.5 \\
\hline 80 & 0.90 & 1649 & 0.15815252 & 0.32398087 & 62 & 77.5 \\
\hline 80 & 0.95 & 2986 & 0.17796620 & 0.36009861 & 61 & 76.2 \\
\hline 150 & 0.75 & 2279 & 0.26194273 & 0.29866150 & 91 & 60.6 \\
\hline 150 & 0.80 & 1273 & 0.20636391 & 0.22933754 & 93 & 62.0 \\
\hline 150 & 0.85 & 3257 & 0.15468909 & 0.27518240 & 94 & 62.6 \\
\hline 150 & 0.90 & 1136 & 0.25482251 & 0.28218144 & 94 & 62.6 \\
\hline 150 & 0.95 & 2452 & 0.25456480 & 0.26788158 & 91 & 60.6 \\
\hline 250 & 0.75 & 3617 & 0.25754282 & 0.31144435 & 120 & 48.0 \\
\hline 250 & 0.80 & 3274 & 0.20844638 & 0.25112189 & 121 & 48.4 \\
\hline 250 & 0.85 & 3066 & 0.15805103 & 0.19299910 & 121 & 48.4 \\
\hline 250 & 0.90 & 2343 & 0.20634355 & 0.20432140 & 121 & 48.4 \\
\hline 250 & 0.95 & 2047 & 0.25541276 & 0.27844937 & 120 & 48.0 \\
\hline
\end{tabular}

rate is 0.03 . Then, for a mutation rate of 0.03 , we analyze the fitness, varying the rate of crossover operator throughout all generations, as shown in Figure 11. Note that in the graph there is a faster fitness convergence for crossover rate equal 0.80 . We conclude that there is a better behaviour of the GA when applying the mutation rate.

In addition, we analyze the incidence of crossover operator on the final results. The Figures 12 show the behavior of the hits average versus crossover rate with very few samples (20), many (80) and many documents (150) respectively.

It makes clear that the GA perform better when using a rate of 0.80 for the crossover operator, regardless of the sample. Therefore, this value appears to be ideal if we maximize the efficiency of the algorithm, concluding that this the rate that gives us better results.

Finally, Table 5 sets out all the parameters that define the behavior of our GA.
Finally, note that using the parameters of the GA which we have determined, the evolution is similar to the one coming out from unsupervised algorithms, the algorithm evolves and looks for groups of related documents with no need of any additional information about the category the documents belong to.

\subsection{Method of Assessing Results}

For the analysis of results, study the following aspects:

1) Effectiveness of the cluster: It is the most important indicator comparing the results to address the quality of the cluster. The values obtained for the "best hits" have been analysed [19] (those who returned the best values in the function of adaptation) and the average values obtained in five runs of the evolutionary algorithm.

2) Evolution of fitness: Analyzing the evolution of fitness in each of the runs, for each of the samples of documents with the aim of determining their behaviour. 
3) Convergence of the algorithm: To ensure the robustness of GA, we analyzed the mean convergence of the algorithm on all executions.

In order to better compare the results, we introduced in the system parameters shown in Table $\mathbf{4}$ for all runs.

In addition, all results are compared with those obtained by the Kmeans supervised clustering algorithm in optimal conditions. Therefore, all experiments that evaluated the quality of the evolutionary algorithm were made in advance knowing the actual results, varying for each test the number of documents to cluster, and taking groups of two known categories of documentary collection. Thus, the results are always compared with real data obtained from the documentary process, being able to verify the efficiency of the GA [20]. In each run all the documents were processed by the evolving system with different seeds (5) that depended on the runtime system. In regard to Kmeans algorithm, it was executed with the same samples processed, passing as data the number of groups is desired, then also better compare the effectiveness the proposed algorithm against its performance in ideal conditions.

\subsection{Better Results with Samples from Reuters Documents in the Collections (Collections in English)}

Analyzed the behaviour for the four distributions of Reuters collection, the tables below (Tables 6 and 7, Tables
8 and 9) show the best result and the average result for each sample.

In the following graphs (Figures 13(a)-(d)), we show in percentage, the efficiency of the GA with different samples of documents in different distributions of the collection documents Reuters.

\subsection{Better Results with Samples of Documents in the Collection of Publishers (Collections in Spanish)}

In Table 10, we perform the same treatment with the

Table 5. Parameters considered for evolutionary system (GA).

\begin{tabular}{cc}
\hline Parameters & Values \\
\hline Population Size (Number of & 50 \\
Trees) & 4000 \\
Generations & 2 \\
Tournament Size & 0.03 \\
Mutation Rate & 0.80 \\
Crossover Rate & Very Few (20), Few (50), Many (80), \\
Number of Documents & Enough Documents (150), and More \\
& Documents (250) \\
Number of Terms Lemmatisers & 40 \\
Coefficient $\alpha$ & 0.85 \\
Threshold Depth & $7 / 10$ \\
\hline
\end{tabular}

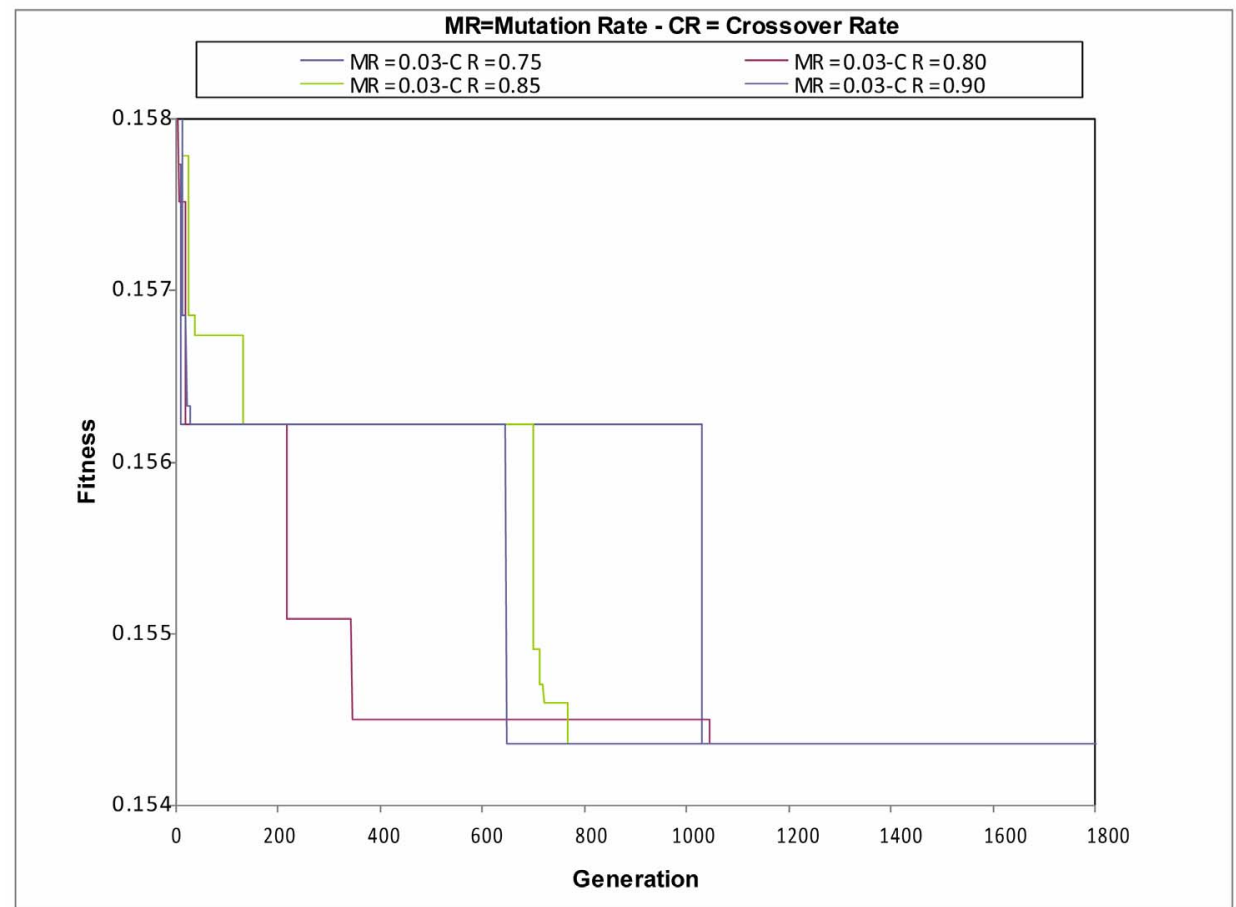

Figure 11. Evolution of fitness by varying the rate of crossover operator GA for a fixed mutation rate equal to 0.03 . It is seen that the values of crossover rate close to 0.80 improves the convergence of the GA. 


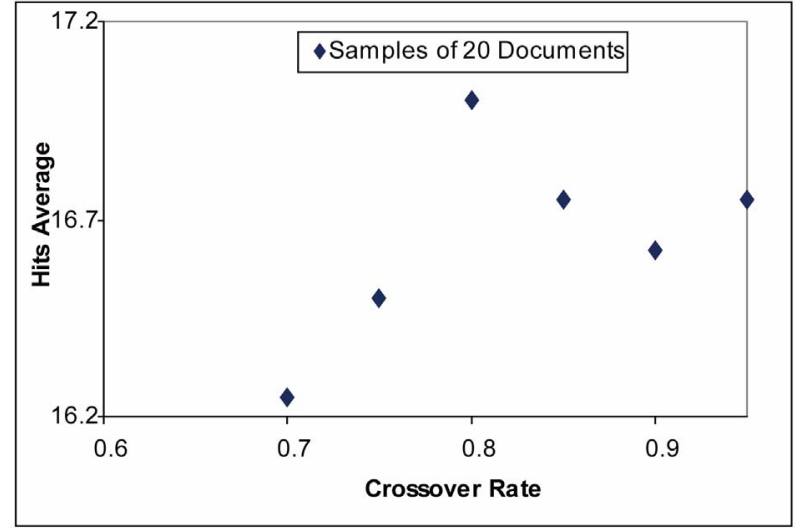

(a)

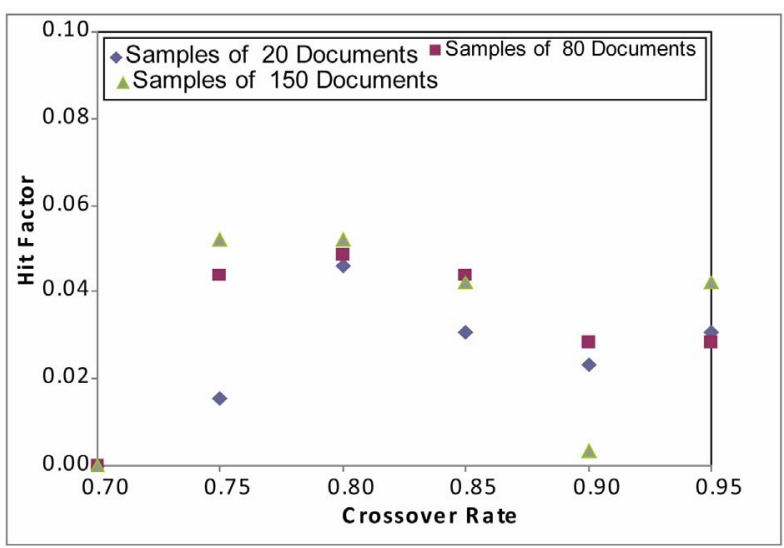

(c)

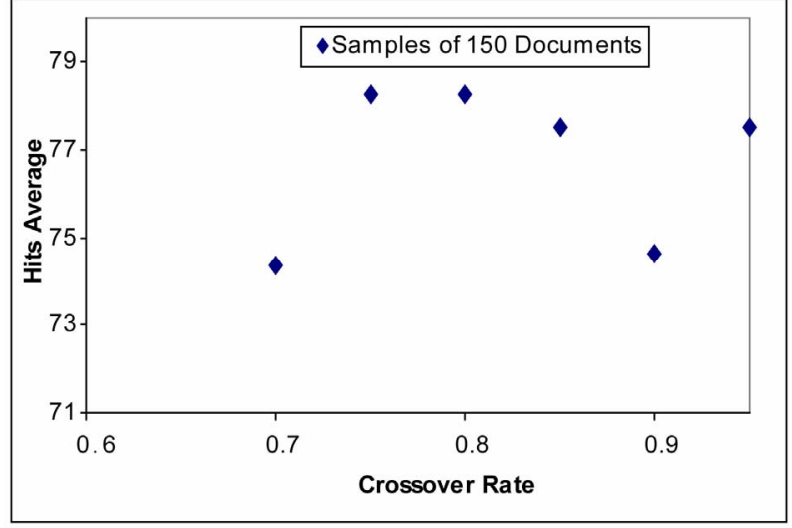

(b)

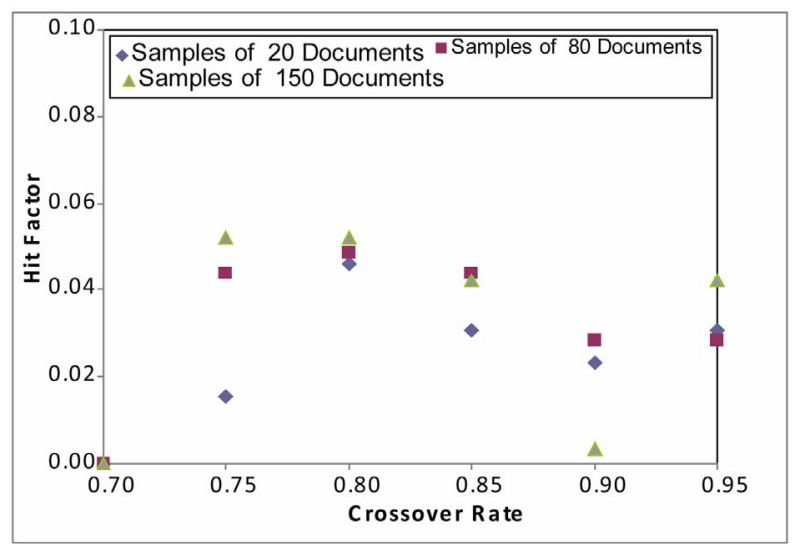

(d)

Figure 12. Hits average of GA with samples 20, 80 and 150 documents varying crossover rate and hit the GA factor against the crossover rates.

Table 6. Comparative results Evolutionary System with various samples of documents showing the best results and the average results of evaluations with the "Distribution 2" of the Reuters 21578 collection.

\begin{tabular}{|c|c|c|c|c|c|c|c|}
\hline \multirow{2}{*}{$\begin{array}{c}\text { Distribution } 2 \text { Reuters } \\
\text { Collection } 1\end{array}$} & \multirow{2}{*}{\multicolumn{3}{|c|}{$\begin{array}{c}\text { Documents Categories: Acq y Earn } \\
\text { Best Result }\end{array}$}} & \multirow{2}{*}{\multicolumn{4}{|c|}{ Best Average }} \\
\hline & & & & & & & \\
\hline Samples of Documents & Fitness & Effectiveness & Convergence & Average Fitness & Deviation Fitness & $\begin{array}{l}\text { Average } \\
\text { Converg }\end{array}$ & Kmeans \\
\hline $\begin{array}{c}\text { Very Few documents ( } 20 \\
\text { documents) }\end{array}$ & 0.155447570 & $85 \%$ (18 hits) & 886 & 0.15545476 & 0.00000828 & 1086 & 16.6 \\
\hline $\begin{array}{l}\text { Few Documents }(50 \\
\text { Documents) }\end{array}$ & 0.156223280 & $94 \%$ (47 hits) & 3051 & 0.15624280 & 0.00002329 & 2641 & 45.8 \\
\hline $\begin{array}{l}\text { Many Documents ( } 80 \\
\text { Documents) }\end{array}$ & 0.159009400 & $89 \%$ (71 hits) & 2500 & 0.15921181 & 0.00020587 & 2246 & 67.8 \\
\hline $\begin{array}{l}\text { Enough Documents (150 } \\
\text { Documents) }\end{array}$ & 0.165013920 & $77 \%$ (115 hits) & 2342 & 0.16508519 & 0.00007452 & 2480 & 121.6 \\
\hline $\begin{array}{l}\text { More Documents ( } 246 \\
\text { Documents) }\end{array}$ & 0.174112100 & $69 \%$ (170 hits) & 2203 & 0.17430502 & 0.00033602 & 2059 & 202.8 \\
\hline
\end{tabular}

Spanish collection of documents from editorials of the "El Mundo" newspaper from the years 2006 and 2007.

The following figures (Figures 14 and 15) show the effectiveness of the GA and the convergence of it.

In the overall, our algorithm gives good results with both documentary collections. We can say that at the end of the evolution and using all the parameters studied and configured for the algorithm, we offer a high effectiveness to perform both clustering of documents. Thus, the algorithm is stable and robust, independently of the sam- 
Table 7. Comparative results Evolutionary System with various samples of documents showing the best results and the average results of evaluations with the "Distribution 8" of the Reuters 21578 collection.

\begin{tabular}{|c|c|c|c|c|c|c|c|}
\hline \multirow{3}{*}{$\begin{array}{c}\text { Distribution } 8 \text { Reuters } \\
\text { Collection } 2 \\
\text { Samples of documents }\end{array}$} & \multirow{2}{*}{\multicolumn{3}{|c|}{$\begin{array}{l}\text { Documents Categories: Acq y Earn Best } \\
\text { Result }\end{array}$}} & \multirow{2}{*}{\multicolumn{4}{|c|}{ Best Average }} \\
\hline & & & & & & & \\
\hline & Fitness & Effectiveness & Convergence & Average Fitness & Deviation Fitness & Average Converg. & Kmeans \\
\hline $\begin{array}{c}\text { Very Few documents (20 } \\
\text { documents) }\end{array}$ & 0.151163560 & $85 \%$ (17 hits) & 555 & 0.15116356 & 0.00000000 & 679 & 15.8 \\
\hline $\begin{array}{l}\text { Few Documents ( } 50 \\
\text { Documents) }\end{array}$ & 0.154856500 & $96 \%$ (48 hits) & 1615 & 0.15485650 & 0.00000000 & 1334 & 43.8 \\
\hline $\begin{array}{l}\text { Many Documents ( } 80 \\
\text { Documents) }\end{array}$ & 0.157073880 & $85 \%$ (68 hits) & 746 & 0.15708362 & 0.00000898 & 1360 & 66.2 \\
\hline $\begin{array}{c}\text { Enough Documentos (150 } \\
\text { Documents) }\end{array}$ & 0.162035070 & $69.3 \%$ (104 hits) & 1989 & 0.16242664 & 0.00033091 & 2283 & 117.6 \\
\hline $\begin{array}{l}\text { More Documents (188 } \\
\text { Documents) }\end{array}$ & 0.163014600 & $68.63 \%$ (129 hits) & 2293 & 0.16334198 & 0.00027325 & 1773 & 140.6 \\
\hline
\end{tabular}

Table 8. Comparative results Evolutionary System with various samples of documents showing the best results and the average results of evaluations with the "Distribution 20" of the Reuters 21578 collection.

\begin{tabular}{|c|c|c|c|c|c|c|c|}
\hline \multirow{2}{*}{\begin{tabular}{|c|} 
Distribution 20 Reuters \\
Collection 3 \\
Samples of Documents
\end{tabular}} & \multicolumn{3}{|c|}{ Documents Categories: Acq y EarnBest Result } & \multicolumn{4}{|c|}{ Best Average } \\
\hline & Fitness & Effectiveness & Convergence & Average Fitness & Deviation Fitness & Average Converg & Kmeans \\
\hline $\begin{array}{c}\text { Very Few Documents (20 } \\
\text { Documents) }\end{array}$ & 0.153027060 & $85 \%$ (17 hits) & 1092 & 0.15321980 & 0.00018398 & 1108 & 16.8 \\
\hline $\begin{array}{l}\text { Few Documents (50 } \\
\text { Documents) }\end{array}$ & 0.156198620 & $92 \%$ (46 hits) & 2173 & 0.15666137 & 0.00030077 & 2635 & 44.8 \\
\hline $\begin{array}{l}\text { Many Documents ( } 80 \\
\text { Documents) }\end{array}$ & 0.158069980 & $81.25 \%$ (65 hits) & 2196 & 0.15810383 & 0.00001884 & 1739 & 66.8 \\
\hline $\begin{array}{c}\text { Enough Documents (108 } \\
\text { Documents) }\end{array}$ & 0.159031080 & $69.4 \%$ (75 hits) & 1437 & 0.15927630 & 0.00026701 & 2636 & 82.2 \\
\hline
\end{tabular}

Table 9. Comparative results Evolutionary System with various samples of documents showing the best results and the average results of evaluations with the "Distribution 21" of the Reuters 21578 collection.

\begin{tabular}{|c|c|c|c|c|c|c|c|}
\hline Distribution 21 Reuters & Documents $\mathrm{C}$ & togrije. Aca & arnBest Result & & Best Aver & rage & \\
\hline Collection 4 & & & & & & & \\
\hline Samples of Documents & Fitness & Effectiveness & Convergence & Average Fitness & Deviation Fitness & Average Converg & Kmeans \\
\hline $\begin{array}{c}\text { Very Few Documents (20 } \\
\text { Documents) }\end{array}$ & 0.152048900 & $90 \%$ (18 hits) & 1163 & 0.15206069 & 0.00001601 & 1165 & 17.8 \\
\hline $\begin{array}{c}\text { Few Documents (50 } \\
\text { Documents) }\end{array}$ & 0.153006650 & $92 \%$ (46 hits) & 2079 & 0.15304887 & 0.00004569 & 2736 & 45.6 \\
\hline $\begin{array}{l}\text { Many Documents ( } 80 \\
\text { Documents) }\end{array}$ & 0.156029510 & $81 \%$ (65 hits) & 2787 & 0.15637693 & 0.00025014 & 2810 & 66.4 \\
\hline $\begin{array}{c}\text { Enough Documents (132 } \\
\text { Documents) }\end{array}$ & 0.157012180 & $70.4 \%$ (93 hits) & 3359 & 0.15720766 & 0.00024132 & 1980 & 98.6 \\
\hline
\end{tabular}

ple or collections of documents used. The hit rate is very high in both collections of documents (having better effectiveness in the collection Reuters). The results of combined fitness, are as good or better than the algorithm Kmeans.

\section{Conclusions}

This work has done an extension of evolutionary algorithms, incorporating an slp data structure that improves the performance of the algorithm in its task of grouping documents. Considering the average results obtained on all samples in each of the used document collections, we see that good results are obtained, and it is feasible to extend and to design new data structures that can withstand the usual genetic operators, in order to use them in optimization problems. This paper has proposed a solution, using an evolutionary system for the problem of clustering documents. The system has been studied in relation to the number of documents to be processed. Experiments show good performance for collections of less than 150 documents, with hits Kmeans above algorithm. Thus, we found that the effectiveness of our algorithm 


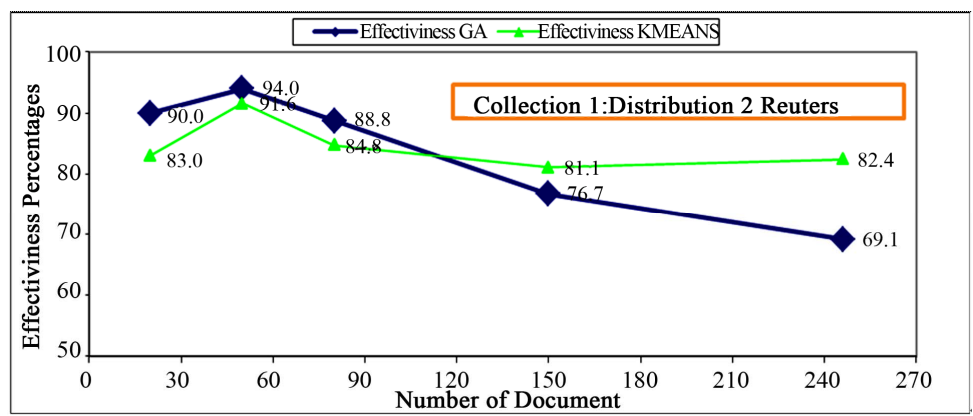

(a)

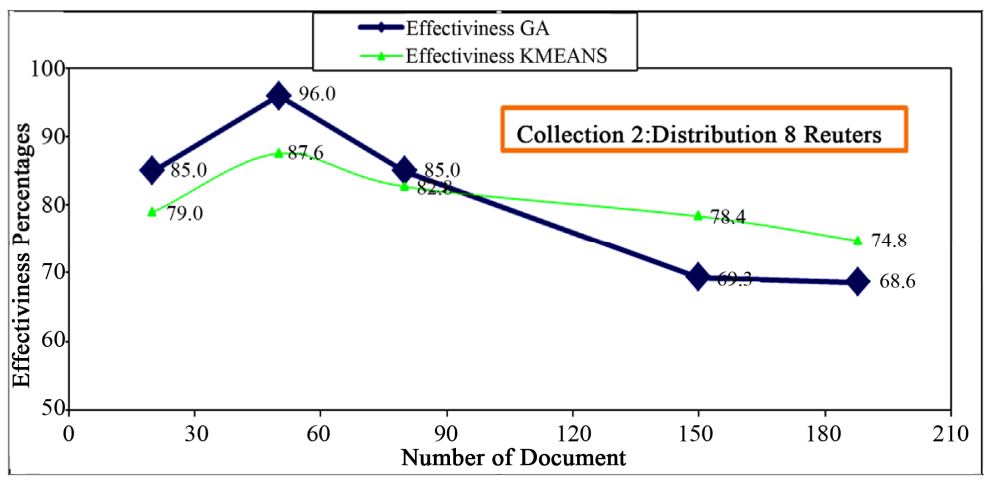

(b)

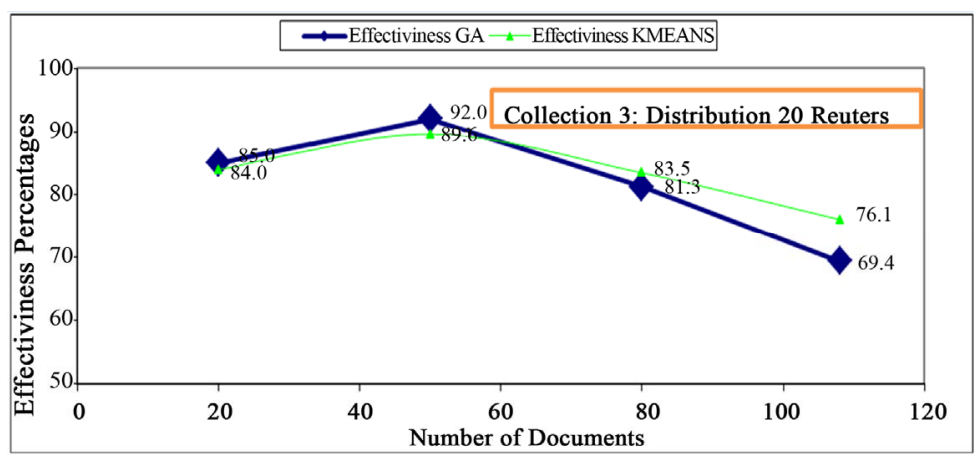

(c)

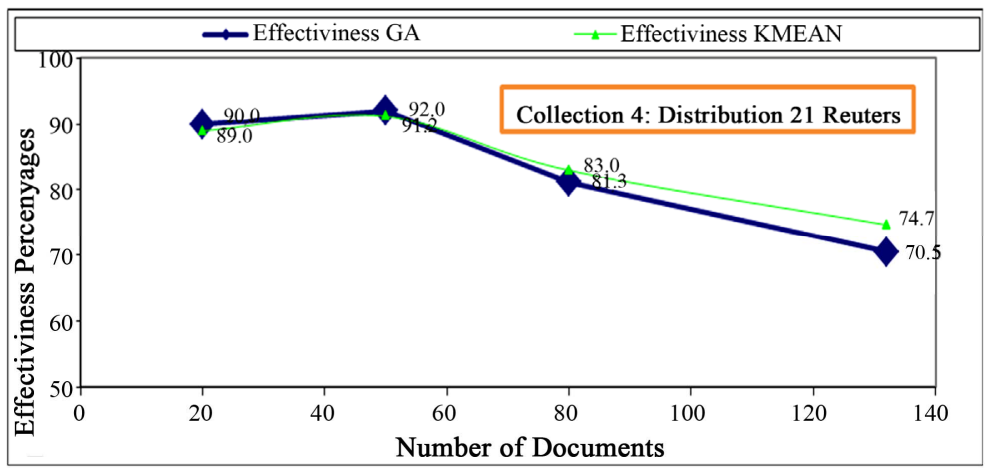

(d)

Figure 13. (a) Comparison of percentages of effectiveness of GA and Kmeans with samples of documents from each of the major distributions of the Reuters collection (Distribution 2); (b) Comparison of percentages of effectiveness of GA and Kmeans with samples of documents from each of the major distributions of the Reuters collection (Distribution 8); (c) Comparison of percentages of effectiveness of GA and Kmeans with samples of documents from each of the major distributions of the Reuters collection (Distribution 20); (d) Comparison of percentages of effectiveness of GA and Kmeans with samples of documents from each of the major distributions of the Reuters collection (Distribution 21). 
Table 10. Comparative results of GA with various samples of documents showing the best results and the average results of evaluations with the "Distribution Editorials in Spanish" from the years 2006 and 2007.

\begin{tabular}{|c|c|c|c|c|c|c|c|}
\hline \multirow{3}{*}{$\begin{array}{c}\text { Documents in Spanish } \\
\text { Editorials } \\
\text { Samples of Documents }\end{array}$} & \multirow{2}{*}{\multicolumn{3}{|c|}{$\begin{array}{c}\text { Documents Categories: Vida Política y } \\
\text { Derecho Best Results }\end{array}$}} & \multirow{2}{*}{\multicolumn{4}{|c|}{ Best Average }} \\
\hline & & & & & & & \\
\hline & Fitness & Effectiviness & Converg. & Average Fitness & Deviation Fitness & Average Converg. & Kmeans \\
\hline $\begin{array}{l}\text { Very Few Documents ( } 20 \\
\text { Documents) }\end{array}$ & 0.152444850 & $85 \%$ (17 hits) & 1026 & 0.15265851 & 0.00012727 & 2196 & 16.6 \\
\hline $\begin{array}{l}\text { Few Documents }(50 \\
\text { Documents) }\end{array}$ & 0.154187850 & $96 \%$ (48 hits) & 2839 & 0.15437233 & 0.00009278 & 2678 & 44.2 \\
\hline $\begin{array}{l}\text { Many Documents ( } 80 \\
\text { Documents) }\end{array}$ & 0.155099730 & $83.7 \%$ (67 hits) & 3137 & 0.15537314 & 0.00026143 & 3085 & 66.8 \\
\hline $\begin{array}{l}\text { Enough Documents (150 } \\
\text { Documents) }\end{array}$ & 0.162184980 & $78.6 \%$ (118 hits) & 1244 & 0.16249632 & 0.00027400 & 1547 & 122.8 \\
\hline $\begin{array}{l}\text { More Documents }(238 \\
\text { Documents) }\end{array}$ & 0.172107860 & $70.1 \%$ (167 hits) & 3387 & 0.17257683 & 0.00028775 & 2021 & 179.6 \\
\hline
\end{tabular}

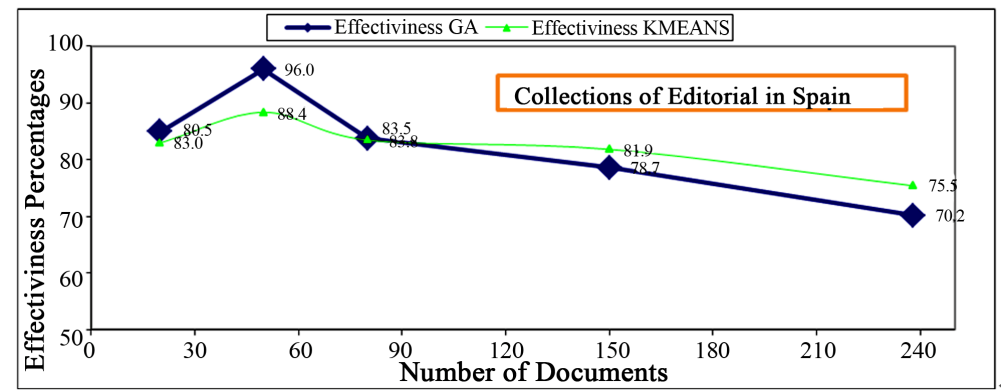

Figure 14. Effectiveness of the GA with the collection of editorials in the Spanish newspaper "El Mundo".

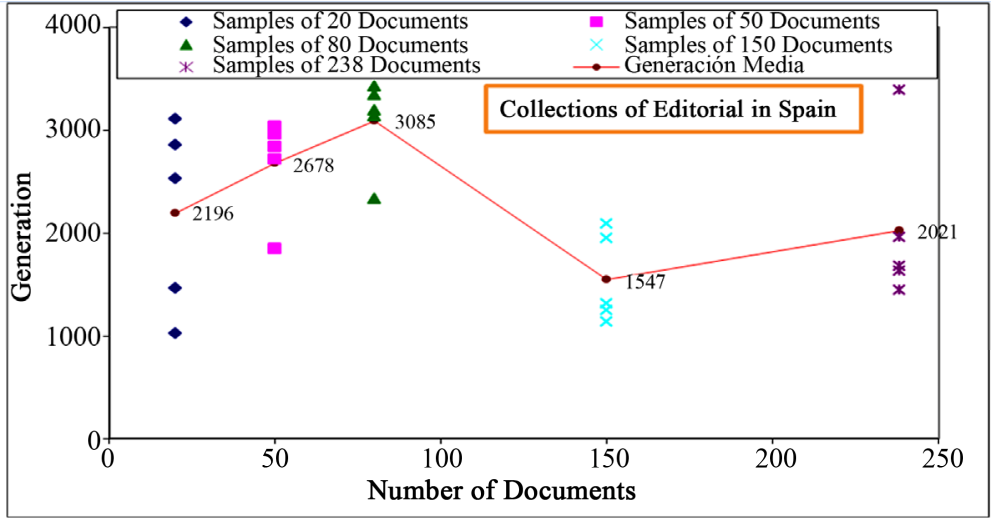

Figure 15. Convergence of the GA with the collection of editorials in the Spanish newspaper "El Mundo".

exceeds Kmeans processing less than 150 documents, and equates this algorithm when processing more than 150 documents, and is therefore an alternative to consider for the grouping of documents with the added advantage that it do so unsupervised. Additionally, the fitness used by our algorithm, shows very little dispersion in all tests with different samples of documents when we use a value of $\alpha$ less than 0.85 , in both document collections (Spanish and English), may be applied by the end user, to facilitate review of consultation documents, after conducting a search, with very little dispersion and unsupervised way.

The genetic algorithm used is a semi supervised algorithm, which requires setting some parameters. The evolution determines the behavior of the algorithm, acting in a flexible manner to adjust rates for operators of the algorithm. For document clustering using a genetic algorithm was used combining two measurement functions (distance and similarity), to better characterize the relationship between the documents and found their proper behavior and we found experimentally that an appropriate value for the parameter $\alpha$, which is involved in the formula that 
combines linearly and inverse distance metric of similarity of our fitness, has to take values close to 0.85 . Exceeded this value, the contribution of both metrics will gradually and cease to be adequate. We used a mutation rate equal to 0.03 along with a crossover rate of 0.80 , to ensure that our results are better than the Kmeans algorithm. Finally, we conclude that using an evolutionary approach with appropriate parameters, is a preferable alternative to traditional methods.

\section{Acknowledgements}

We would like to thank Departament of Computer Science of University of Alcalá for its support and for providing this research oportunity.

Work done under project: VOA3Rr: Virtual Open Access Agriculture \& Aquaculture Repository: Sharing Scientific and Scholarly Research related to Agriculture, Food, and Environment (CIP-ICT-PSP.2009.2.4: 250525VOA-3R)

\section{REFERENCES}

[1] R. Baeza-Yates and B. Ribeiro Neto, "Modern Information Retrieval," 2nd Edition, ACM Press Book AddisonWesley, New York, 1999.

[2] Z. Michalewicz, "Genetic Algorithms + Data Structures = Evolution Program," Third Edition, Springer-Verlag, Berlin, 1999.

[3] M. Aldaz, J. Heintz, G. Matera, J. L. Montaña and L. M. Pardo, "Time-Space Tradeoffs in Algebraic Complexity Theory," Journal of Complexity, Vol. 16, No. 1, 1998, pp. 2-49. doi:10.1006/jcom.1999.0526

[4] C. L. Alonso, J. L. Montaña and J. Puente, "Straight Line Programs: A New Linear Genetic Programming Approach," Proceedings of the 20th International Conference of the IEEE on Tools with Artificial Intelligence, ICTAI, Dayton, 3-5 November 2008, pp. 517-524.

[5] D. Goldberg, "Genetic Algorithms in Search, Optimization and Machine Learning," First Edition, AddisonWesley, 1989.

[6] J. Koza, "Genetic Programming: On the Programming Computers by Means of Natural Selection," Massachusetts Institute of Technology, Cambridge, 1992.

[7] J. L. Castillo, J. R. Fernández del Castillo and L. González, "Feature Reduction for Document Clustering with NZIPF Method," Proceedings of IADIS International Conference e-Society 2009, Barcelona, 25-28 February 2009, pp. 205-209.

[8] J. L. Castillo, J. R. Fernández del Castillo and L. Gon- zález, "Methodology of Preprocessing of Documents for Systems of Recovery of Information," Proceedings of IADIS International Conference, Information System 2008, Algarve, 9-11 April 2008, pp. 324-327.

[9] G. K. Zipf, "Human Behavior and the Principle of Least Effort: An Introduction to Human Ecology," Addison Wesley, Cambridge, 1949.

[10] J. A. Moreiro González, “Applications from Content Analysis of the Mathematical Theory of Information," Journal of Documentation, Vol. 5, 2002, pp. 273-286.

[11] M. L. Pao, "Automatic Indexing Based on Goffman Transition of Word Occurrences," American society for Information Science. Meeting 40th Information Management in the 1980's: Proceedings of the ASIS Annual Meeting, Chicago, 26 September-1 October 1977.

[12] M. Berry and M. Castellanos, "Survey of Text Mining II,” Springer Verlag, London, 2008. doi:10.1007/978-1-84800-046-9

[13] J. Arabas, Z. Michalewicz Zbigniew and J. Mulawka "GAVaPs - A Genetic Algorithm with Varying Population Size," Proceedings of the First Conference on Evolutionary Computation of the IEEE, orlando, 27-29 June 1994, pp. 73-79.

[14] J. L. Castillo, J. R. Fernández del Castillo and L. González, "Information Retrieval with Cluster Genetic," Proceedings of IADIS International Conference on Data Mining, Amsterdam, 24-26 July 2008, pp. 77-81.

[15] J. L. Castillo, J. R. Fernández del Castillo and L. González, "Group Method of Documentary Collections using Genetic Algorithms," Proceedings Part II of Distributed Computing, Artificial Intelligence, Bioinformatics, Soft Computing, and Ambient Assisted Living. 10th International Work-Conference on Artificial Neural Network IWANN, Salamanca, 10-12 June 2009, pp. 992-1000.

[16] L. Davis, "Reuters-21578 Text Categorization Test collection," 1987.

http://www.daviddlewis.com/resources/testcollections/reu ters $21578 /$

[17] Eurovoc, "Eurovoc Thesaurus. Alphabetic Presentation Permuted," Edition 4.2, Spanish Language, European Communities, 2006.

[18] D. Olson and D. Delen, “Advanced Data Mining Techniques," Springer-Verlag, Berlin, 2008.

[19] A. M. Robertson and P. Willet, "Generation of Equifrecuent Groups of Words Using a Genetic Algorithms," Journal of Documentation, Vol. 50, No. 3, 1994, pp. 213 232.

[20] J. C. Bezdeck, "Genetic Algorithms Guided Clustering," IEEE Proceedings of the First Conference on Evolutionary Computation, Orlando, 27-29 June 1994, pp. 34-40. 